\title{
SINERGIA
}

\author{
REVISTA DO INSTITUTO DE CIÊNCIAS ECONÔMICAS, ADMINISTRATIVAS E CONTÁBEIS (ICEAC)
}

\section{DETERMINANTES DO TEMPO MÉDIO DE SUBSTITUIÇÕES NO FUTEBOL: UMA ANÁLISE DO CAMPEONATO BRASILEIRO DE 2014}

\author{
CLÁUDIO DJISSEY SHIKIDA \\ ARI FRANCISCO DE ARAÚJO JÚNIOR* \\ RAFAEL CANUTO NEVES
}

\begin{abstract}
RESUMO
Este estudo analisa os padrões da primeira substituição de jogadores de cada time durante o intervalo ou o segundo tempo de jogos na primeira divisão do Campeonato Brasileiro de 2014. Foram usados modelos MQO (Mínimos Quadrados Ordinários) e Tobit, ambos com correção para heterocedasticidade. Os resultados mostram que times que estão ganhando tendem a fazer a primeira mudança nos momentos finais da partida. Ademais, substituições neutras, seguidas das ofensivas e, apenas, posteriormente, as defensivas são utilizadas pelos técnicos, em média. Também existem evidências de que a classificação no campeonato de ambas as equipes em campo tem influência no momento em que a alteração ocorre.
\end{abstract}

Palavras-chave: substituições; futebol; campeonato brasileiro.

\section{ABSTRACT}

This paper analyses the patterns on the first player substitution of each team during half-time or the second half of the match. It is based on data of first division of the Brazilian Championship of 2014. There were used OLS (Ordinary Least Squares) and Tobit regressions, both with correction for heteroskedasticity. The results show that the winning team tends to delay its first substitution. Therefore, offensive substitutions occur before defensive ones. There is also evidence that the team table position also influences the moment that substitutions happens.

Key-words: substitutions; soccer; Brazilian soccer championship.

Recebido em: 25-03-2019 Aceito em: 17-06-2019

\section{INTRODUÇÃO}

Uma partida de futebol é jogada em dois tempos de quarenta e cinco minutos mais os minutos de acréscimo entre dois times de onze jogadores. Os jogadores são, geralmente, classificados em quatro áreas de atuação diferentes dentro de campo: goleiro, defensores, meio-campistas e atacantes. Com participação ativa em uma partida de futebol, o comandante da equipe tem a função de organizar e reorganizar o elenco ao longo de uma partida, observando o desempenho passado, presente ou possíveis situações futuras da mesma. Essas mudanças podem ocorrer para manter ou aumentar uma possível vantagem no placar, produzir a recuperação de um resultado ruim ou, mesmo, testar jogadores ou esquemas táticos.

Um dos mais importantes recursos de um técnico de futebol é realizar substituições dos jogadores ao longo da partida. Essas substituições podem ser feitas com vários objetivos, tal como evitar um excesso de fadiga, evitar uma expulsão, apenas por motivos táticos. Três é número máximo de trocas de jogadores que um técnico pode realizar em uma partida.

Este trabalho tem como objetivo analisar os fatores que determinam as substituições no futebol, tendo como base dados da série A do Campeonato Brasileiro do ano de 2014, especificamente o tempo decorrido entre o começo do segundo tempo de jogo e a decisão do treinador de alterar a equipe inicial. Neste estudo, foi levada em consideração apenas a primeira troca de jogadores, exceto trocas envolvendo os goleiros.

Como a grande maioria das substituições feitas no primeiro tempo são causadas por fatores fora do controle do técnico, como expulsões e contusões, são consideradas apenas as mudanças feitas no intervalo

\footnotetext{
"Doutor em Economia pela Universidade Federal do Rio Grande do Sul. Mestre em Economia pela Universidade São Paulo. Graduado em Ciências Econômicas pela Universidade Federal de Minas Gerais. E-mail: claudio.shikida@ufpel.edu.br

".."Mestre em Teoria Econômica pela Universidade Federal de Minas Gerais. Graduado em Economia pela Universidade São Paulo.

"** Bacharel em Economia pela Universidade Federal de Pelotas
} 
e segundo tempo de jogo. Portanto, este estudo analisa apenas os dados da primeira substituição de jogadores de linha de uma equipe quando realizada do intervalo até o final das partidas do Campeonato Brasileiro de Futebol (Série A) de 2014.

\section{REVISÃO DA LITERATURA}

Este trabalho se inspira no artigo publicado por Corral, Barros e Prieto-Rodriguez (2008). Os autores analisam possíveis estratégias do tempo de substituição de jogadores em uma partida de futebol na temporada 2004-2005 da primeira divisão do Campeonato Espanhol. Os resultados sugerem que o fator mais relevante é o placar do jogo. Outro determinante apontado é o mando de campo, ou seja, times jogando em casa tendem a fazer mudanças mais rapidamente, possivelmente respondendo à pressão da torcida.

Outro estudo importante foi de Myers (2012). O artigo analisa dados das ligas italiana, espanhola, alemã, americana, além de informações da Copa do Mundo de Futebol de 2010. O autor propõe momentos ótimos para a realização das substituições como otimização de um recurso escasso por serem possíveis apenas três. Concluiu-se que os melhores momentos para a troca de jogadores são aos 13, 28 e 34 minutos do segundo tempo, quando não houve contusões ou expulsões no primeiro tempo.

Seguindo este mesmo foco de pesquisa, Silva e Swartz (2016), utilizando metodologia distinta, sugerem resultados um tanto diferentes daqueles de Myers (2012) sobre os melhores momentos da partida para realizar as substituições. Além disso, em uma partida entre times de desempenho semelhante, o time com uma desvantagem no placar é o mais provável a marcar um gol no segundo tempo. Isso se deve ao fato de que o time com a vantagem no placar tende a mandar menos jogadores ao ataque em uma tentativa de segurar o resultado atual, proporcionando, potencialmente, mais oportunidades ao adversário.

Coelho et al. (2012) analisa o efeito das substituições sobre a intensidade do jogo no segundo tempo de uma partida de futebol. Foi utilizada a variável "frequência cardíaca" como medida de esforço dos jogadores em campo como medida para a intensidade de uma equipe da Série A do campeonato brasileiro. Os autores concluem que, mesmo ocorrendo uma queda de ímpeto no segundo tempo, esta é atenuada pelo efeito das mudanças na equipe durante o intervalo. Os autores separaram o campo em áreas de menor e maior intensidade e, a partir daí, observaram, em média, que os jogadores ficam mais tempo nas zonas de maior intensidade quando ocorrem as substituições.

Outros estudos relacionados a substituições valem ser comentados. Um estudo qualitativo da experiência vivida pelos jogadores reservas em comparação com os titulares da liga inglesa foi realizado por Woods e Thatcher (2009). Os autores concluíram que a titularidade influencia fortemente 0 aspecto psicológico dos atletas. Em outras palavras, a falta de comunicação adequada por parte do técnico e/ou do psicólogo da equipe gera um relacionamento conturbado entre o substituto e o titular de sua posição e, até mesmo, com o próprio treinador.

Existem também estudos que analisam a situação do técnico ao longo da partida. Um deles foi realizado por Hirotsu e Wright (2006) sobre mudanças na formação tática de um time na liga japonesa. Por meio de um modelo markoviano, analisou-se a propensão de um time marcar ou sofrer gols e a propensão de se ganhar ou perder a posse da bola nas partidas. Foram encontradas evidências de que, independentemente da estratégia do time com mando de campo, o time visitante sempre utilizará a tática que lhe dá a menor probabilidade de derrota, e não a tática que maximize a probabilidade de vitória.

Explicitada a relevância do problema na literatura, a seção seguinte detalha a base de dados utilizada.

\section{BASE DE DADOS}

Este estudo utiliza dados da primeira divisão do Campeonato Brasileiro de 2014. O campeonato é composto de vinte times que se enfrentam duas vezes ao longo do ano, em turno e returno e, desta maneira, todas as equipes têm trinta e oito partidas na temporada. O resultado final da partida determina quantos pontos a equipa ganhará na rodada em questão. O time vencedor acumula mais 3 (três) pontos; caso haja empate; ambos os times ganham 1 (um) ponto, e a equipe perdedora não acumula nenhum ponto adicional.

A base de dados analisa todas as partidas da temporada e suas 754 substituições ao longo dos jogos que são pertinentes para este estudo. Todos os dados foram coletados a partir das súmulas feitas pelos árbitros após cada partida e da tabela de classificação do campeonato, ambas disponíveis no site oficial da CBF (Confederação Brasileira de Futebol) e no de estatísticas e dados futebolísticos, Futpedia, pertencente à Rede Globo.

O motivo das substituições (lesão, fadiga ou tática) não é especificado nas fontes de dados. Assim, foram desconsideradas as mudanças de goleiro e as de outros jogadores no primeiro tempo, que, 
geralmente, são utilizadas para minimizar o efeito de lesões ou situações inesperadas de jogo sobre o desempenho da equipe. Afinal, essas substituições não representariam exatamente uma escolha estratégica do técnico e seriam induzidas por motivos como contusões e/ou expulsões. Assim, foram utilizadas apenas a primeira substituição feita por cada time durante o intervalo ou o segundo tempo de jogo. O tempo em minutos até a substituição é a variável analisada.

Neste estudo, foram consideradas quatro variáveis explicativas, cuja escolha é inspirada em Corral, Barros e Prieto-Rodriguez (2008). A primeira é o efeito do mando de campo sobre a estratégia de substituição de atletas. Para representar esta categoria, foi utilizada uma dummy de mando de campo ("casa"). Esta variável toma o valor de 1 (um), caso o time, realizando a substituição, seja o time da casa, e, de 0 (zero), em caso contrário.

Outra dimensão avaliada tenta capturar alteração de atleta relacionada a algum tipo de mudança tática adotada pelo treinador da equipe. São três variáveis dummies: "ofens" (a saída de um defensor para a entrada de um atacante, por exemplo, que $29,08 \%$ das mudanças), "neutra" (troca entre jogadores da mesma posição, representando $51,39 \%$ das mudanças) e "defens" (a saída de um atacante para a entrada de um defensor, por exemplo, representando $19,52 \%$ das mudanças). Tais variáveis dummies tentam capturar o caráter da substituição, isto é, a intenção do técnico em avançar (ofens), manter (neutra) ou recuar (defens) a organização da equipe.

Avalia-se, também, o efeito do placar parcial da partida no momento em que a substituição ocorre. Esta variável "placar" é calculada como a diferença entre os gols marcados e os subtraídos, ou seja, caso positivo, o time que faz a mudança está ganhando o jogo. Caso seja negativo, o time está perdendo (e quando a variável assume o valor zero, temos que o jogo está empatado).

Finalmente, levamos em consideração um grupo de variáveis que controlam a qualidade dos dois times em campo. Para esta categoria, foram coletadas duas informações adicionais sobre as equipes. As variáveis ordinais "posic" e "posicr" representam, respectivamente, a posição do time realizando a substituição e de seu adversário na classificação do campeonato antes da partida. Ambas podem variar entre 1 (primeira posição no campeonato antes da partida) a 20 (vigésima posição no campeonato antes da partida).

Uma análise inicial dos dados sugere uma diferença em relação ao observado por Corral, Barros e Prieto-Rodriguez (2008). Para os autores, o mando de campo é um fator importante, porque os times mandantes tendem a fazer as substituições no intervalo para evitar a pressão da torcida. Segundo os dados coletados para a Série A do Campeonato Brasileiro, temporada de 2014, das 754 substituições, 245 foram feitas no intervalo e apenas $44,49 \%$ delas foram realizadas pelo time da casa. Isso representa apenas $28,91 \%$ das substituições de times mandantes.

Adicionalmente, $52,71 \%$ das substituições feitas por times com saldo negativo na partida têm caráter ofensivo, enquanto, nos times, com vantagem no placar, $35,83 \%$ das mudanças são defensivas, e $50,83 \%$ são neutras. Também de acordo com as conclusões de Myers (2012), observa-se que, em média, a primeira substituição foi feita aos 13,09 minutos do segundo tempo da partida do jogo. Claro que, como apresentado na Figura 1, o intervalo de jogo é a moda amostral, isto é, o período em que os treinadores mais frequentemente optaram para realizar as mudanças.

\section{METODOLOGIA}

Neste estudo, estimamos modelos para a primeira substituição de cada time a partir do intervalo em cada partida do Campeonato Brasileiro de 2014. A variável dependente é o tempo em minutos decorridos entre o começo do segundo tempo de jogo e a decisão do treinador de alterar a equipe inicial. $O$ valor da variável é zero para alterações no intervalo da partida. 
FIGURA 1 - Frequência de Substituições no Intervalo e por Minuto do Segundo Tempo

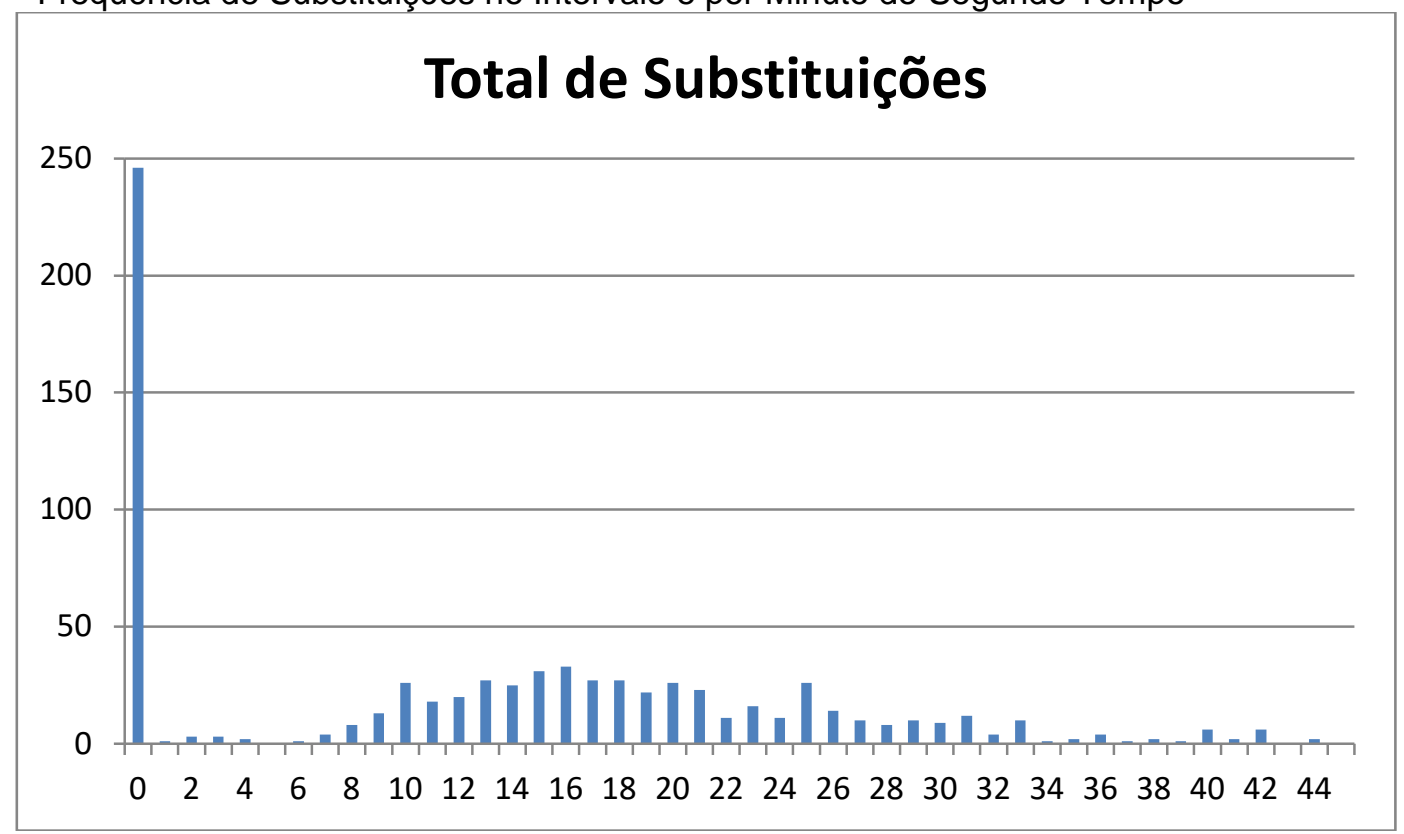

A elevada frequência de zeros na variável dependente, ilustrada na Figura 1, implica que o uso de modelos de regressão com base no algoritmo de Mínimos Quadrados Ordinários (MQO) é inadequado por conta do potencial viés gerado. De qualquer forma, são apresentados os resultados por MQO para fins de interpretação dos coeficientes, com erros-padrão "sanduíche" (White) para correção de heterocedasticidade.

O elevado número de zeros na variável dependente indica que a distribuição desta é "censurada à esquerda" (left-censored) e o modelo mais adequado é o Tobit. Temos um número significativo de substituições que acontecem no minuto zero (com probabilidade elevada), e o restante das mesmas ocorrem ao longo do segundo tempo de jogo. A estimação de um modelo via Mínimos Quadrados Ordinários levaria a resultados não consistentes, já que a amostra censurada é não representativa da população (Cameron e Trivedi, 2009).

Sua estimação leva em conta que y (dependente) é uma variável observada de uma variável latente (não observada) $\mathrm{y}^{\star}$ (desejo de substituir um atleta),tal que:

$$
\begin{aligned}
& y^{*}=\beta_{0}+x \beta+u \quad u \mid x \sim \text { Normal }\left(0, \sigma^{2}\right) \\
& y=\max \left(0, y^{*}\right)
\end{aligned}
$$

No caso deste artigo, o vetor $x$ inclui as variáveis "Placar", "Posic", "Posicr", "Ofens", "Neutro" e "Defens" anteriormente explicadas. Os parâmetros são estimados por meio da maximização da log-verossimilhança. Neste caso, eles podem ser interpretados como efeitos marginais das explicativas sobre a latente $y^{*}$.

Para nossos propósitos, a interpretação dos sinais dos parâmetros estimados é o suficiente.

Para testar a significância do conjunto de parâmetros desta regressão, realizamos o teste de razão de verossimilhança, ou LR (Likelihood Ratio). Além disso, as estatísticas dos testes individuais dos parâmetros, neste caso, seguem uma distribuição normal padrão.

Vale notar que, de acordo com a construção das variáveis do vetor $\mathrm{x}$, valores positivos dos coeficientes implicam adiamento das substituições, isto é, o treinador opta por manter o time titular mais tempo em campo. Consequentemente, valores negativos nos coeficientes implicam adiantamento da mudança. Em qualquer das situações, caso seja adicionada mais uma unidade na variável explicativa ou, em caso de uma dummy, se ela tiver o valor igual a 1.

\section{RESULTADOS}

Os resultados são apresentados a seguir. Vale notar que, de fato, a variável mando de campo (casa) não foi significativa estatisticamente ao nível de $10 \%$ (veja Tabela A no Anexo). Dessa forma, as variáveis explicativas consideradas são placar, posic, posicr, ofens, neutro e defens. A Tabela 1 apresenta os resultados do modelo estimado por MQO, e a Tabela 2, o modelo estimado por Tobit. 
TABELA 1 - Resultados da regressão em MQO

\begin{tabular}{l|l|l}
\hline Min & Coef. & $P>|t|$ \\
\hline Placar & 2.764611 & 0.000 \\
\hline Posic & -0.2580463 & 0.000 \\
\hline Posicr & -0.1151066 & 0.087 \\
\hline Ofens & -4.579712 & 0.000 \\
\hline Neutro & -5.135385 & 0.000 \\
\hline Defens & -4.049208 & 0.000 \\
\hline Teste F & \multicolumn{2}{|c}{$F(6,748)=15.74$} \\
\end{tabular}

FONTE: Elaboração dos Autores.

TABELA 2 - Resultado regressão em Tobit

\begin{tabular}{l|l|l}
\hline Min & Coef. & $P>|t|$ \\
\hline Placar & 4.057901 & 0.000 \\
\hline Posic & -0.3398374 & 0.001 \\
\hline Posicr & -0.1890986 & 0.051 \\
\hline Ofens & -6.30361 & 0.000 \\
\hline Neutro & -7.089532 & 0.000 \\
\hline Defens & -6.381434 & 0.000 \\
\hline
\end{tabular}

FONTE: Elaboração dos autores.

A Tabela 1 mostra que os parâmetros estimados são significativos individual e conjuntamente (testes "t" e "F").

Além disso, os resultados sugerem que o placar tem uma influência positiva no minuto de jogo em que a substituição ocorre. Pode-se concluir que, em média, um placar favorável adia a substituição de um jogador (no intervalo e/ou no segundo tempo) em 2,77 minutos. Isso, possivelmente, está relacionado ao fato de o técnico da equipe optar por manter o time titular em campo por mais tempo, dado que este se mostra capaz de sustentar um resultado de vitória sobre o adversário. Isso corrobora a expressão popular "não se mexe em time que está ganhando" e com a conclusão Corral, Barros e Prieto-Rodriguez (2008).

Quanto à estimativa do efeito da qualidade das equipes em campo, observa-se um efeito negativo sobre o tempo de substituição. Vale ressaltar novamente que um aumento nestas variáveis indica uma piora na qualidade da equipe. As variáveis "posic" e "posicr" mostram que o desempenho da equipe até a partida induz a uma antecipação da primeira substituição da equipe (no intervalo e/ou no segundo tempo). A piora de uma posição no ranking faz com que, na partida seguinte, a substituição (no intervalo e/ou no segundo tempo) ocorra 0,26 minutos antes, em média.

No caso da classificação da equipe adversária, observa-se uma relação similar. Quanto melhor classificada a equipe rival no campeonato, a mudança ocorre, aproximadamente, 0,12 minutos antes. Para Corral, Barros e Prieto-Rodriguez (2008), a qualidade do time que promove a substituição não é variável estatisticamente significativa.

Analisando os coeficientes estimados das dummies de mudança tática realizada pelo treinador, observa-se uma relação negativa com o minuto da substituição e um comportamento um tanto conservador dos técnicos. Ao comparar quantitativamente os efeitos marginais, pode-se concluir que as alterações de caráter neutro tendem a ocorrer antes, seguidas pelas ofensivas e, por último, pelas defensivas. Apesar do estudo de Corral, Barros e Prieto-Rodriguez (2008) apresentar um efeito marginal positivo nas substituições defensivas, a discrepância entre os valores dos coeficientes neste trabalho mostra que as mudanças de caráter defensivo tendem a ocorrer mais tarde.

Os resultados acima são confirmados na estimação Tobit (também corrigida para heterocedasticidade). $O$ modelo é significativo estatisticamente $(L R=49,83$ e valor $-p=0)$. Todas as variáveis mantêm-se significativas individualmente ao nível de 10\% (Tabela 3). Os resultados qualitativos são iguais aos estimados por MQO. Ou seja, os sinais dos efeitos esperados são os mesmos, independentemente do processo de estimação escolhido. 


\section{CONCLUSÃO}

O trabalho analisou os determinantes do tempo médio das substituições de jogadores nas partidas de futebol da Série A do Campeonato Brasileiro, temporada 2014, no primeiro e no segundo tempo. Para isso, foram usados dois tipos de regressão: MQO e Tobit, ambos com correção para heterocedasticidade. Os dados mostram uma frequência maior de substituições durante o intervalo.

Os resultados indicam que a variável que exerce a maior influência quantitativa sobre o minuto em que a mudança corre é o placar parcial da partida. Além disso, a posição de ambos os times envolvidos também é um fator significativo. A escolha tática da substituição realizada pelo treinador também apresenta resultado interessante, já que, em média, alterações de caráter neutro tendem a ocorrer antes, seguidas pelas ofensivas e, por último, por aquelas de caráter defensivo.

\section{REFERÊNCIAS}

BARROS, Carlos Pestana; FRICK, Bernd; PASSOS, José. Coaching for Survival: The Hazards of Head Coach Careers in the German "Bundesliga". Applied Economics, Taylor \& Francis (Routledge), 2009.

CAMERON, Colin, A; Trivedi, Pravin, K. Microeconometrics Using Stata. Stata Press Publication, 2009.

COELHO, Daniel Barbosa; COELHO, Leonardo Gomes Martins; MORANDI, Rodrigo Figueiredo; JUNIOR, João Batista Ferreira; MARINS, João Carlos Bouzas; PRADO, Luciano Sales; SOARES, Danusa Dias; SILAMI-GARCIA, Emerson. Effect of Player Substitutions on the Intensity of Second-Half Soccer Match Play. Revista Brasileira de Cineantropometria e Desempenho Humano, vol. 14, no. 2, Florianópolis, 2012.

DEL CORRAL, Julio; BARROS, Carlos Pestana; PIETRO-RODRÍGUEZ, Juan. The Determinants of Soccer Player Substitutions: A Survival Analysis of the Spanish Soccer League. Journal of Sports Economics, 2008.

KONING, Ruud. An Econometric Evaluation of the Effect of Firing a Coach on Team Performance. University of Groningen/ UMCG research database, 2003.

MYERS, Bret. A Proposed Decision Rule for the Timing of Soccer Substitutions. Journal of Quantitative Analysis in Sports, 2012.

SILVA, Rajitha; SWARTZ, Tim. Analysis of Substitution Times in Soccer. Journal of Quantitative Analysis in Sports, 2016.

WOODS, Bernadette; TATCHER, Joanne.A Quantitative Exploration of Substitutes' Experiences in Soccer. The Sports Psychologist, 2009.

WRIGHT, Mike; HIROTSU, Nibuyoshi. Modeling Tactical Changes of Formation in Association Football as a ZeroSum Game. Journal of Quantitative Analysis in Sports vol.2, 2006. 


\section{ANEXO}

TABELA A - Resultados da regressão em MQO, com todas as variáveis explicativas (inclusive casa)

\begin{tabular}{l|l|l}
\hline Min & Coeficiente & $P>|t|$ \\
\hline Casa & 0.0369501 & 0.976 \\
\hline Placar & 4.052385 & 0.000 \\
\hline Posic & -0.3401282 & 0.001 \\
\hline Posicr & -0.1887865 & 0.054 \\
\hline Ofens & -6.29341 & 0.000 \\
\hline Neutro & -7.076509 & 0.000 \\
\hline Defens & -6.368409 & 0.000 \\
\hline
\end{tabular}


